\title{
Partition of portal-drained visceral net flux in beef steers
}

\author{
2. Net flux of volatile fatty acids, $D$ - $\beta$-hydroxybutyrate and L-lactate across \\ stomach and post-stomach tissues*
}

\author{
BY CHRISTOPHER K. REYNOLDS AND GERALD B. HUNTINGTON \\ U.S. Department of Agriculture, Agricultural Research Service, Ruminant Nutrition \\ Laboratory, Building 162, BARC-East, Beltsville, MD 20705, USA and University of \\ Maryland, College Park, MD 20742, USA
}

(Received 8 March 1988 - Accepted 23 May 1988)

\begin{abstract}
1. Net flux of volatile fatty acids (VFA), D- $\beta$-hyroxybutyrate (BOHB) and L-lactate across post-stomach (anterior mesenteric-drained viscera (MDV)), stomach and total hepatic portal-drained viscera (PDV) tissues was measured in two beef steers (mean live weight $390 \mathrm{~kg}$ ).

2. Net flux was measured while steers were fed, in sequence, on (1) chopped lucerne (Medicato sativa) (twelve meals/d), (2) chopped lucerne (two meals/d) and (3) a pelleted concentrate diet containing $780 \mathrm{~g}$ ground maize/ $\mathrm{kg}$ (two meals/d).

3. Five hourly net flux measurements were obtained on $2 \mathrm{~d}$ for each dietary regimen, beginning $0.5 \mathrm{~h}$ before a meal delivered at 08.00 hours. Net flux was calculated as venous-arterial concentration differences (VA) multiplied by blood flow (measured by downstream dilution of $p$-aminohippurate (PAH)).

4. Stomach tissues accounted for 85 to over $100 \%$ of net VFA and BOHB appearance across PDV. Net appearance across stomach tissues represented $74 \%$ of net PDV L-lactate appearance.

5. When lucerne was given, there was net utilization of arterial acetate and BOHB across MDV. This MDV utilization may reflect acetate and BOHB use as an energy source or their incorporation into mesenteric fat.

6. When concentrate was given, more $n$-butyrate and $n$-valerate and less L-lactate appeared across PDV and less 3-methylbutyrate appeared across stomach tissues than when lucerne was given. Postprandial increases in VFA, $\mathrm{BOHB}$ and L-lactate net flux across PDV followed meal-feeding of lucerne.

7. On a net basis, the relative contribution of MDV tissues to total PDV net appearance of VFA and BOHB was small $(<15 \%)$ in these steers.
\end{abstract}

In ruminants, much of the carbon oxidized to meet energy requirements and expired as carbon dioxide is initially assimilated from the diet as a component of volatile fatty acids (VFA). These VFA are the products of microbial fermentation of carbohydrates in the lumen of the gut. Calculations of Bergman \& Wolff (1971) show that substantial quantities of acetate, propionate and butyrate produced in the rumen of sheep do not appear in the portal blood, presumably due to their metabolism by rumen epithelial tissues. The ketone bodies $\mathrm{D}$ - $\beta$-hydroxybutyrate (BOHB) and acetoacetate are products of the metabolism of butyrate and, to a lesser extent, acetate by tissues of the portal-drained viscera (PDV) (Stevens \& Stettler, 1966) and L-lactate is a product of the metabolism of absorbed propionate by rumen epithelium (Giesecke \& Stangassinger, 1980).

In the classic study of Barcroft et al. (1944), concentrations of VFA in blood draining sections of the gastrointestinal tract of anaesthetized sheep were compared with arterial VFA concentrations. The substantial venous-arterial concentration differences (VA) measured for the rumen, reticulum and, to a lesser extent, the omasum and caecum, led to the conclusion that significant amounts of VFA were absorbed from the gut into the portal blood. Since this pioneering research there have been few, if any, studies comparing relative amounts of VFA absorption across various sections of the ruminant PDV. We know of no studies in which measurements of net VFA flux have been obtained simultaneously from sections of the PDV of sheep or cattle.

* Scientific article no. A4668 and contribution no. 7664 of the University of Maryland. 
The anatomy of the PDV provides an opportunity for the venous return from the gastrointestinal tract to be separated into primarily stomach and post-stomach portions. The objective of our study was to compare net flux of VFA, BOHB and L-lactate across stomach and post-stomach tissues of beef steers fed on lucerne (Medicago sativa) or concentrate diets.

\section{MATERIALS AND METHODS}

Surgical procedures, dietary regimens and sampling protocol

Implantation of chronic catheters, dietary protocol and sampling procedures have been described (Reynolds \& Huntington, 1988). Concisely, catheters were implanted using asceptic surgical techniques into the hepatic portal, anterior mesenteric, gastrosplenic and distal mesenteric veins and the caudal aorta of two Hereford beef steers (mean body weight, $390 \mathrm{~kg}$ ). VA for VFA, BOHB and L-lactate were determined across the total PDV (tissues drained by the hepatic portal vein including the gastrointestinal tract, pancreas, spleen and mesenteric fat), the anterior mesenteric-drained viscera (MDV) or post-stomach tissues and the gastrosplenic-drained viscera or stomach tissues. Blood flow in the anterior mesenteric and hepatic portal veins was determined by measuring the downstream dilution of $p$-aminohippurate (PAH; $90 \mathrm{~g} / \mathrm{kg})$ infused continuously $(1.2 \mathrm{ml} / \mathrm{min})$ into a distal mesenteric vein. A priming dose $(15 \mathrm{ml})$ of $\mathrm{PAH}$ was used to speed the equilibration of PAH concentration.

Steers were fed on chopped lucerne hay (maximum stem length $40 \mathrm{~mm}$ ) before and following surgery using an automatic feeder which delivered $500 \mathrm{~g}$ hay every $2 \mathrm{~h}$ and $50 \mathrm{~g}$ trace mineralized salt (TMS) at 08.00 hours. Five measurements of net flux were obtained at hourly intervals 7 and $9 \mathrm{~d}$ after surgery (timed-fed lucerne).

Steers were then given $3.0 \mathrm{~kg}$ of the same chopped lucerne hay at 08.00 and 16.00 hours and $50 \mathrm{~g}$ TMS at 08.00 hours until measurements of net flux were obtained $21-24 \mathrm{~d}$ after surgery (meal-fed lucerne). After these measurements, steers were adapted over $12 \mathrm{~d}$ to a pelleted concentrate diet (Reynolds \& Huntington, 1988), which was then given in equal meals $(3.0 \mathrm{~kg})$ at 08.00 and 16.00 hours. Measurements of net flux were obtained 28 and $30 \mathrm{~d}$ after the diet change began (meal-fed concentrate).

\section{Sample analyses}

Samples were kept on ice until analyses or treatment before freezing was completed, which was never longer than $3 \mathrm{~h}$ after sampling. Analyses of whole blood and plasma PAH and calculation of blood and plasma flow rates have been described (Reynolds \& Huntington, 1988). Concentrations of L-lactate in fresh plasma were determined using a Yellow Springs Lactate Analyzer (Yellow Springs Instruments Co. Inc., Yellow Springs, OH). Portions of whole blood were deproteinized with $3.48 \mathrm{M}$-perchlorate, which was removed by precipitation with $6 \mathrm{M}$-potassium hydroxide, resulting in $\mathrm{pH}>10$. Following centrifugation at $9000 \mathrm{~g}$, the resulting supernatant fraction was stored frozen until BOHB concentrations were determined using the enzymic procedures of Williamson \& Mellanby (1974).

Portions of whole blood were stored frozen until analysed for VFA concentration using procedures of Reynolds et al. (1986). Briefly, the concentrations of acetate, propionate, $n$ butyrate, isobutyrate, 2-methylbutyrate, 3-methylbutyrate and $n$-valerate in this solution were determined by gas-liquid chromatography of blood samples deproteinized using procedures of Somogyi (1945) and passed through ion-exchange resins.

\section{Statistical analyses}

Calculations of net flux and statistical analyses performed on these values have been described (Reynolds \& Huntington, 1988). Briefly, overall means were generated for each 
steer and dietary regimen and used to test differences between timed- and meal-fed lucerne and concentrate feeding. Overall means for each sampling time $(n 2)$ within each steer and diet were also produced and analysed to determine effects of time and surface responses within each dietary regimen. Orthogonal polynomials were used to test for linear, quadratic, cubic and quartic responses when time effects were significant $(P<0 \cdot 1)$. Stomach net flux rates presented were calculated as PDV minus MDV net flux (Reynolds \& Huntington, 1988).

RESULTS

$V F A$

With the exception of $n$-butyrate, arterial concentrations (Table 1) of $\mathrm{C}_{4}$ and $\mathrm{C}_{5}$ VFA were extremely small. Only arterial concentrations of 3-methylbutyrate were affected by dietary regimen, being higher $(P<0.05)$ when steers were fed on concentrate. Arterial concentrations of acetate increased linearly $(P<0.01)$ when lucerne was meal-fed (Table 2).

Comparison of net flux rates of VFA across MDV and PDV (Table 1) suggests the poststomach tissues make a relatively minor contribution to net VFA absorption across the total PDV. Net flux rates (Table 1) for the VFA across stomach tissues were substantial and on a net basis accounted for nearly all the net absorption of VFA across PDV.

Measurements of VA and net flux of VFA across MDV were not affected $(P>0 \cdot 1)$ by dietary regimen (Table 1). When lucerne was given, net flux of acetate across MDV was negative, indicating that use of arterial acetate was greater than acetate absorption from the gut lumen. Overall, net absorption of VFA across the MDV (excluding acetate) represented from 8 to $15 \%$ of net PDV absorption. Net $n$-butyrate absorption across MDV increased $(P<0.01)$ following meal-feeding of lucerne (Table 2$)$.

Across the PDV, VA for isobutyrate and 3-methylbutyrate were lower $(P<0.05$ and $P<0.1$ respectively), while VA for $n$-butyrate were greater $(\mathrm{P}<0 \cdot 05)$ when concentrate was given than when lucerne was given. Ratios of net PDV absorption of acetate: propionate averaged $3 \cdot 2: 1$, ranging from $2 \cdot 5: 1$ when concentrate was given to $3 \cdot 5: 1$ when lucerne was meal-fed (Table 1).

Across stomach tissues, VA were greater $(P<0.05)$ for $n$-butyrate when concentrate was given than when lucerne was given. Stomach tissue VA for 3-methylbutyrate were greater $(P<0 \cdot 1)$ when lucerne was timed-fed than when lucerne was meal-fed. Net flux of 3methylbutyrate across stomach tissue was lower $(P<0.05)$ when concentrate was given than when lucerne was given (Table 1).

There were some fluctuations $(P<0.05)$ in 2- and 3-methylbutyrate absorption across PDV and stomach tissues when lucerne was timed-fed (values not shown) and when concentrate was given (Table 3 ). Net absorption of $n$-butyrate across PDV and stomach tissue also increased slightly $(P<0.05)$ following meal-feeding of concentrate (Table 3$)$. When lucerne was meal-fed there were significant postprandial increases in net absorption across PDV or stomach tissues, or both, for all the VFA measured except $n$-valerate (Table 2). These responses were generally linear or quadratic, or both $(P<0.05)$.

\section{$B O H B$}

When steers were meal-fed concentrate, arterial concentrations of BOHB (Table 1) were higher $(P<0.01)$ than when lucerne was given. However, dietary regimen had no effect $(P>0 \cdot 1)$ on VA or net flux rates of BOHB across MDV, PDV or stomach tissues (Table 1). Across MDV, net flux of BOHB (Table 1) followed trends for acetate, being negative 
Table 1. Arterial concentration and metabolism of blood volatile fatty acids, $D-\beta$ hydroxybutyrate $(\mathrm{BOHB})$ and L-lactate across portal-drained viscera of two beef steers

\begin{tabular}{|c|c|c|c|c|c|}
\hline Diet... & $\begin{array}{c}\text { Time-fed } \\
\text { lucerne } \\
\text { (Medicago } \\
\text { sativa) }\end{array}$ & $\begin{array}{l}\text { Meal-fed } \\
\text { lucerne }\end{array}$ & $\begin{array}{c}\text { Meal-fed } \\
\text { concentrate }\end{array}$ & Mean & SEM \\
\hline \multicolumn{6}{|c|}{ Arterial concentration (mM) } \\
\hline Acetate & $1 \cdot 352$ & 1.435 & 1.073 & $1 \cdot 287$ & $0 \cdot 167$ \\
\hline Propionate & 0.028 & 0.031 & 0.033 & 0.031 & 0.004 \\
\hline Isobutyrate & $0 \cdot 000$ & 0.001 & 0.000 & 0.001 & 0.000 \\
\hline$n$-Butyrate & $0 \cdot 016$ & 0.008 & 0.031 & 0.018 & 0.005 \\
\hline 2-Methylbutyrate & $0 \cdot 000$ & $0 \cdot 001$ & 0.000 & 0.000 & 0.000 \\
\hline 3-Methylbutyrate & $0 \cdot 000$ & 0.003 & $0.009^{*}$ & 0.004 & $0 \cdot 00 \mathrm{l}$ \\
\hline$n$-Valerate & $0 \cdot 002$ & $0 \cdot 003$ & 0.003 & 0.002 & 0.001 \\
\hline $\mathrm{BOHB}$ & 0.424 & 0.456 & $0.838^{*}$ & 0.572 & 0.026 \\
\hline L-Lactate & $0.363 \ddagger$ & 0.526 & $0 \cdot 371$ & 0.420 & 0.027 \\
\hline \multicolumn{6}{|c|}{ Mesenteric-drained viscera flux $(\mathrm{mmol} / \mathrm{h})$} \\
\hline Acetate & -50 & -26 & 3 & -24 & 21 \\
\hline Propionate & 9 & 10 & 24 & 15 & 4 \\
\hline Isobutyrate & $1 \cdot 5$ & $1 \cdot 7$ & $1 \cdot 6$ & 1.6 & $0 \cdot 3$ \\
\hline$n$-Butyrate & $-0 \cdot 1$ & $2 \cdot 2$ & $12 \cdot 3$ & $4 \cdot 8$ & $2 \cdot 5$ \\
\hline 2-Methylbutyrate & 0.4 & 0.6 & $1 \cdot 2$ & 0.7 & 0.2 \\
\hline 3-Methylbutyrate & $0 \cdot 5$ & $0 \cdot 4$ & 0.5 & $0 \cdot 5$ & 02 \\
\hline$n$-Valerate & $1 \cdot 0$ & 1.8 & $3 \cdot 6$ & $2 \cdot 1$ & 0.6 \\
\hline BOHB & $-3 \cdot 5$ & $-5 \cdot 8$ & $0 \cdot 2$ & $-3 \cdot 0$ & $1 \cdot 1$ \\
\hline L-Lactate & $15 \cdot 0$ & $9 \cdot 8$ & 14.9 & $13 \cdot 2$ & $2 \cdot 1$ \\
\hline \multicolumn{6}{|c|}{ Portal-drained viscera flux $(\mathrm{mmol} / \mathrm{h})$} \\
\hline Acetate & 586 & 610 & 448 & 548 & 77 \\
\hline Propionate & 166 & 174 & 178 & 173 & 33 \\
\hline Isobutyrate & $12 \cdot 7$ & $15 \cdot 2$ & $10 \cdot 5$ & $12 \cdot 8$ & 0.9 \\
\hline n-Butyrate & $31 \cdot 4$ & $31 \cdot 2$ & $92 \cdot 7^{*}$ & $51 \cdot 8$ & $10 \cdot 2$ \\
\hline 2-Methylbutyrate & $7 \cdot 7$ & $10 \cdot 8$ & $12 \cdot 4$ & $10 \cdot 3$ & $1 \cdot 2$ \\
\hline 3-Methylbutyrate & $7 \cdot 9$ & $6 \cdot 5$ & $2 \cdot 0$ & $5 \cdot 5$ & $1 \cdot 2$ \\
\hline$n$-Valerate & $11 \cdot 6$ & $13 \cdot 4$ & $18 \cdot 1^{*}$ & $14-4$ & $0 \cdot 9$ \\
\hline $\mathrm{BOHB}$ & 669 & $70 \cdot 4$ & $105 \cdot 6$ & 80.9 & $15 \cdot 5$ \\
\hline L-Lactate & $53 \cdot 9$ & $55 \cdot 7$ & $43 \cdot 1^{*}$ & 50.9 & $2 \cdot 1$ \\
\hline \multicolumn{6}{|c|}{ Stomach flux $(\mathrm{mmol} / \mathrm{h})$} \\
\hline Acetate & 636 & 636 & 444 & 572 & 98 \\
\hline Propionate & 156 & 164 & 154 & 158 & 36 \\
\hline Isobutyrate & $11 \cdot 2$ & $13 \cdot 5$ & 8.9 & $11 \cdot 2$ & $1 \cdot 2$ \\
\hline$n$-Butyrate & $31 \cdot 5$ & $29 \cdot 0$ & $80 \cdot 4$ & $47 \cdot 0$ & $12 \cdot 2$ \\
\hline 2-Methylbutyrate & $7 \cdot 3$ & $10 \cdot 2$ & $11 \cdot 3$ & $9 \cdot 6$ & $1 \cdot 0$ \\
\hline 3-Methylbutyrate & $7 \cdot 4$ & $6 \cdot 1$ & $1 \cdot 5^{*}$ & $5 \cdot 0$ & 0.9 \\
\hline$n$-Valerate & $10 \cdot 6$ & $11 \cdot 6$ & $14 \cdot 6$ & $12 \cdot 3$ & $1 \cdot 4$ \\
\hline BOHB & $70 \cdot 4$ & $76 \cdot 3$ & $105 \cdot 4$ & $84 \cdot 0$ & $16 \cdot 2$ \\
\hline L-Lactate & 38.9 & $45 \cdot 9$ & $28 \cdot 2$ & $37 \cdot 7$ & $4 \cdot 1$ \\
\hline
\end{tabular}

Significantly different from time-fed or meal-fed lucerne: ${ }^{*} P<0 \cdot 05$.

Significantly different from meal-fed lucerne: $\ddagger P<0 \cdot 05$.

when lucerne was given and essentially zero when concentrate was given, indicating net stomach flux accounted for all net BOHB appearance across PDV tissues (Table 1). When lucerne was timed-fed (values not shown), slight fluctuations occurred in arterial BOHB concentrations (linear $P<0.01$, quadratic $P<0.05$, quartic $P<0.01$ ). Following mealfeeding of lucerne, postprandial increases (Table 2) were measured for arterial concentration (linear $P<0.05$ ), PDV production (linear $P<0.05$ ) and stomach production 
Table 2. Arterial concentration and metabolism of blood volatile fatty acids, $D-\beta$ hydroxybutyrate $(B O H B)$ and L-lactate across portal-drained viscera (PDV) of two beef steers meal-fed lucerne (Medicago sativa) $\dagger$

(Mean values for four determinations)

\begin{tabular}{|c|c|c|c|c|c|c|}
\hline Sampling time (hours) ... & $07 \cdot 30$ & $08 \cdot 30$ & $09 \cdot 30$ & $10 \cdot 30$ & $11 \cdot 30$ & SEM \\
\hline \multicolumn{7}{|l|}{ Blood flow $(1 / \mathrm{h})$} \\
\hline MDV & 196 & 322 & 285 & 282 & 242 & 26 \\
\hline $\operatorname{PDV}^{a} \cdot b$ & 542 & 653 & 697 & 695 & 700 & 16 \\
\hline \multicolumn{7}{|c|}{ Arterial concentration (mM) } \\
\hline Acetate $^{\mathrm{a}}$ & $0 \cdot 778$ & $1 \cdot 270$ & $1 \cdot 595$ & $1 \cdot 748$ & $1 \cdot 784$ & 0.155 \\
\hline Propionate & $0 \cdot 021$ & 0.031 & 0.033 & 0.037 & 0.031 & 0.004 \\
\hline Isobutyrate & 0.002 & 0.001 & 0.001 & 0.001 & 0.001 & 0.001 \\
\hline n-Butyrate & 0.003 & 0.004 & 0.010 & 0.011 & 0.012 & 0.003 \\
\hline 2-Methylbutyrate & 0.001 & 0.001 & 0.001 & 0.001 & 0.001 & 0.001 \\
\hline 3-Methylbutyrate & 0.006 & 0.001 & 0.004 & 0.002 & 0.003 & 0.003 \\
\hline$n$-Valerate & 0.002 & 0.002 & 0.005 & 0.003 & 0.003 & 0.002 \\
\hline BOHB $^{\mathbf{a}, \mathrm{b}}$ & $0 \cdot 323$ & 0.414 & 0.491 & 0.511 & 0.539 & 0.021 \\
\hline L-Lactate ${ }^{\mathbf{a}, \mathbf{b}}$ & $0 \cdot 379$ & 0.499 & $0 \cdot 618$ & 0.569 & 0.566 & $0 \cdot 024$ \\
\hline \multicolumn{7}{|l|}{ MDV flux (mmol/h) } \\
\hline Acetate & 13 & -23 & -50 & -18 & -50 & 24 \\
\hline Propionate & 6 & 18 & 11 & 10 & 7 & 5 \\
\hline Isobutyrate & $1 \cdot 3$ & $2 \cdot 6$ & $1 \cdot 8$ & $2 \cdot 2$ & $0 \cdot 7$ & 0.5 \\
\hline$n$-Butytrate ${ }^{\mathrm{a}}$ & $2 \cdot 0$ & $4 \cdot 1$ & $2 \cdot 1$ & $2 \cdot 6$ & 02 & $1 \cdot 0$ \\
\hline 2-Methylbutyrate & 0.6 & 1.4 & 0.6 & 0.6 & $0 \cdot 1$ & $0 \cdot 3$ \\
\hline 3-Methylbutyrate & $-0 \cdot 3$ & 1.6 & $0 \cdot 1$ & $0 \cdot 7$ & -0.1 & $0 \cdot 6$ \\
\hline$n$-Valerate & 0.9 & $4 \cdot 342$ & $1 \cdot 0$ & 1.7 & $1 \cdot 1$ & $1 \cdot 4$ \\
\hline ВОНВ & $-1 \cdot 4$ & $-7 \cdot 4$ & -7.4 & $-6 \cdot 5$ & $-6 \cdot 5$ & 1.7 \\
\hline L-Lactate & $3 \cdot 1$ & $18 \cdot 4$ & $11 \cdot 9$ & $8 \cdot 2$ & $7 \cdot 4$ & $6 \cdot 3$ \\
\hline \multicolumn{7}{|l|}{ PDV flux $(\mathrm{mmol} / \mathrm{h})$} \\
\hline Acetate $^{a, b}$ & 349 & 522 & 681 & 743 & 757 & 39 \\
\hline Propionate ${ }^{u, b}$ & 71 & 168 & 209 & 209 & 214 & 19 \\
\hline Isobutyrate ${ }^{a, b}$ & $10 \cdot 4$ & $17 \cdot 2$ & $17 \cdot 2$ & 16.4 & 14.9 & $1 \cdot 1$ \\
\hline$n$-Butyrate ${ }^{\mathrm{a}}$ & $11 \cdot 6$ & $25 \cdot 2$ & $32 \cdot 2$ & $43 \cdot 5$ & $43 \cdot 4$ & $3 \cdot 7$ \\
\hline 2-Methylbutyrate & 6.9 & $13 \cdot 2$ & $13 \cdot 3$ & $11 \cdot 2$ & $9 \cdot 7$ & $1 \cdot 3$ \\
\hline 3-Methylbutyrate & 0.9 & 7.9 & 8.9 & $8 \cdot 5$ & $6 \cdot 2$ & $2 \cdot 8$ \\
\hline$n$-Valerate & $4 \cdot 1$ & $12 \cdot 0$ & $15 \cdot 2$ & $18 \cdot 3$ & $17 \cdot 5$ & $3 \cdot 7$ \\
\hline $\mathrm{BOHB}^{\mathrm{a}}$ & $47 \cdot 9$ & $71 \cdot 9$ & $69 \cdot 0$ & $81 \cdot 2$ & $81 \cdot 9$ & $4 \cdot 8$ \\
\hline L-Lactate & $27 \cdot 2$ & $62 \cdot 3$ & $74 \cdot 4$ & $59 \cdot 6$ & $55 \cdot 0$ & 68 \\
\hline \multicolumn{7}{|l|}{ Stomach flux $(\mathrm{mmol} / \mathrm{h})$} \\
\hline Acetate $e^{a, b}$ & 336 & 545 & 731 & 761 & 807 & 48 \\
\hline Propionate $^{\mathrm{a}, \mathrm{b}}$ & 65 & 150 & 197 & 199 & 207 & 21 \\
\hline Isobutyrate ${ }^{a, b}$ & $9 \cdot 1$ & 14.6 & $15 \cdot 4$ & $14 \cdot 2$ & $14 \cdot 2$ & $1 \cdot 2$ \\
\hline$n$-Butyrate ${ }^{a}$ & 9.6 & $21 \cdot 1$ & $30 \cdot 3$ & $40 \cdot 9$ & $43 \cdot 2$ & $4 \cdot 3$ \\
\hline 2-Methylbutyrate ${ }^{\mathrm{b}}$ & $6 \cdot 3$ & 11.8 & $12 \cdot 7$ & $10 \cdot 6$ & $9 \cdot 6$ & $1 \cdot 2$ \\
\hline 3-Methylbutyrate ${ }^{a, b}$ & $1 \cdot 1$ & $6 \cdot 3$ & $8 \cdot 8$ & $7 \cdot 8$ & $6 \cdot 4$ & $2 \cdot 4$ \\
\hline$n$-Valerate & $3 \cdot 2$ & $7 \cdot 7$ & $14 \cdot 1$ & $16 \cdot 7$ & $16 \cdot 4$ & $3 \cdot 6$ \\
\hline $\mathrm{BOHB}^{\mathrm{a}, \mathrm{b}}$ & $49 \cdot 3$ & $79 \cdot 3$ & $76 \cdot 5$ & $87 \cdot 6$ & $88 \cdot 4$ & $4 \cdot 1$ \\
\hline L-Lactate & $24 \cdot 1$ & $43 \cdot 9$ & $62 \cdot 5$ & $51 \cdot 4$ & $47 \cdot 6$ & $8 \cdot 4$ \\
\hline
\end{tabular}

MDV, mesenteric-drained viscera.

a, b Linear and quadratic response respectively; $P<0.05$.

$\dagger 3.0 \mathrm{~kg}$ chopped lucerne hay at 08.00 and 16.00 hours. 
Table 3. Arterial concentration and metabolism of blood volatile fatty acids, $D-\beta$ hydroxybutyrate $(\mathrm{BOHB})$ and L-lactate across portal-drained viscera (PDV) of two beef steers meal-fed concentrate $\dagger$

(Mean values for four determinations)

\begin{tabular}{|c|c|c|c|c|c|c|}
\hline Sampling time (hours)... & 07.30 & 08.30 & 09.30 & 10.30 & 11.30 & SEM \\
\hline \multicolumn{7}{|l|}{ Blood flow $(\mathrm{l} / \mathrm{h})$} \\
\hline MDV & 333 & 340 & 373 & 235 & 234 & 57 \\
\hline PDV & 808 & 762 & 756 & 710 & 692 & 41 \\
\hline \multicolumn{7}{|c|}{ Arterial concentration (mM) } \\
\hline Acetate & 1.027 & 1.098 & $1 \cdot 080$ & $1 \cdot 116$ & $1 \cdot 044$ & 0.077 \\
\hline Propionate & 0.032 & 0.033 & 0.033 & 0.036 & 0.032 & 0.004 \\
\hline Isobutyrate & 0.002 & 0.001 & $0 \cdot 00 \mathrm{I}$ & $0 \cdot 001$ & 0.001 & 0.001 \\
\hline$n$-Butyrate & 0.028 & $0 \cdot 038$ & 0.026 & 0.036 & 0.034 & 0.007 \\
\hline 2-Methylbutyrate & 0.001 & $0 \cdot 001$ & 0.001 & 0.001 & 0.001 & 0.001 \\
\hline 3-Methylbutyrate & 0.004 & 0.015 & 0.014 & 0.004 & 0.009 & 0.004 \\
\hline$n$-Valerate & 0.002 & 0.007 & $0 \cdot 004$ & 0.002 & 0.009 & 0.004 \\
\hline ВOHB & 0.788 & 0.821 & 0.811 & 0.898 & 0.872 & 0.032 \\
\hline L-Lactate & 0.352 & $0 \cdot 384$ & $0 \cdot 381$ & $0 \cdot 374$ & $0 \cdot 365$ & 0.008 \\
\hline \multicolumn{7}{|l|}{ MDV flux (mmol/h) } \\
\hline Acetate & 14 & 7 & -14 & 12 & -4 & 11 \\
\hline Propionate & 29 & 25 & 23 & 23 & 17 & 6 \\
\hline Isobutyrate & $1 \cdot 5$ & 1.9 & $1 \cdot 9$ & 1.4 & 12 & 03 \\
\hline$n$-Butyrate & 16.0 & $12 \cdot 1$ & $12 \cdot 6$ & $9 \cdot 2$ & $7 \cdot 3$ & $3 \cdot 8$ \\
\hline 2-Methylbutyrate & 1.0 & $1 \cdot 8$ & $1 \cdot 2$ & 0.9 & 0.7 & $0 \cdot 3$ \\
\hline 3-Methylbutyrate & $1 \cdot 0$ & $-0 \cdot 8$ & 0.4 & $3 \cdot 2$ & -0.5 & 0.3 \\
\hline$n$-Valerate & $4 \cdot 1$ & $3 \cdot 4$ & 3.6 & $2 \cdot 5$ & $2 \cdot 8$ & 1.6 \\
\hline ВOHB & $-3 \cdot 6$ & $-10 \cdot 7$ & $-6 \cdot 2$ & $-10 \cdot 6$ & $28 \cdot 2$ & $17 \cdot 3$ \\
\hline L-Lactate & $15 \cdot 4$ & $18 \cdot 1$ & $15 \cdot 4$ & $10 \cdot 6$ & $46 \cdot 1$ & $12 \cdot 5$ \\
\hline \multicolumn{7}{|l|}{ PDV flux $(\mathrm{mmol} / \mathrm{h})$} \\
\hline Acetate & 409 & 452 & 495 & 458 & 435 & 45 \\
\hline Propionate & 185 & 190 & 180 & 173 & 157 & 7 \\
\hline Isobutyrate & $10 \cdot 0$ & $12 \cdot 3$ & $11 \cdot 4$ & $9 \cdot 8$ & $8 \cdot 8$ & $1 \cdot 2$ \\
\hline$n$-Butyrate & $100 \cdot 6$ & $106 \cdot 5$ & 96.7 & $77 \cdot 9$ & $68 \cdot 0$ & 49 \\
\hline 2-Methylbutyrate & $13 \cdot 5$ & $15 \cdot 5$ & $9 \cdot 8$ & $12 \cdot 4$ & $10 \cdot 5$ & $2 \cdot 1$ \\
\hline 3-Methylbutyrate & $3 \cdot 9$ & $4 \cdot 7$ & 0.5 & 18 & $1 \cdot 8$ & $2 \cdot 4$ \\
\hline$n$-Valerate & $20 \cdot 1$ & $17 \cdot 2$ & $20 \cdot 4$ & $15 \cdot 6$ & $14 \cdot 1$ & $4 \cdot 2$ \\
\hline BOHB & $106 \cdot 2$ & $95 \cdot 1$ & $149 \cdot 5$ & $85 \cdot 5$ & 67.6 & $24 \cdot 5$ \\
\hline L-Lactate & $42 \cdot 5$ & $46 \cdot 0$ & $42 \cdot 1$ & $42 \cdot 9$ & $43 \cdot 2$ & $2 \cdot 8$ \\
\hline \multicolumn{7}{|l|}{ Stomach flux $(\mathrm{mmol} / \mathrm{h})$} \\
\hline Acetate & 396 & 445 & 510 & 446 & 440 & 38 \\
\hline Propionate & 156 & I64 & 157 & 150 & 139 & 12 \\
\hline Isobutyrate & 8.5 & $10 \cdot 4$ & 9.6 & 8.5 & 7.7 & $1 \cdot 2$ \\
\hline$n$-Butyrate ${ }^{a, b}$ & $84 \cdot 6$ & $94 \cdot 3$ & $84 \cdot 1$ & $68 \cdot 7$ & $60 \cdot 7$ & $2 \cdot 9$ \\
\hline 2-Methylbutyrate & $12 \cdot 5$ & 13.6 & $8 \cdot 6$ & $11 \cdot 5$ & $9 \cdot 8$ & 1.8 \\
\hline 3-Methylbutyrate & $2 \cdot 9$ & $5 \cdot 5$ & $0 \cdot 1$ & $-1 \cdot 3$ & $-1 \cdot 3$ & $2 \cdot 2$ \\
\hline$n$-Valerate & $16 \cdot 0$ & $13 \cdot 8$ & $16 \cdot 8$ & $13 \cdot 2$ & $11 \cdot 2$ & 3.8 \\
\hline BOHB $^{b}$ & $109 \cdot 7$ & $105 \cdot 8$ & $155 \cdot 7$ & $96 \cdot 2$ & $39 \cdot 4$ & $20 \cdot 1$ \\
\hline L-Lactate & $27 \cdot 1$ & $27 \cdot 9$ & $26 \cdot 7$ & $31 \cdot 3$ & -2.9 & 9.8 \\
\hline
\end{tabular}

MDV, mesenteric-drained viscera.

${ }^{\mathrm{a}, \mathrm{b}}$ Linear and quadratic response respectively: $P<0.05$.

$\dagger 3.0 \mathrm{~kg}$ concentrate at 08.00 and 16.00 hours. 
of BOHB (linear $P<0.05$, quadratic $P<0.05$ ). A similar increase in stomach production of BOHB (quadratic $P<0.05$ ) followed meal-feeding of concentrate (Table 3 ).

\section{L-lactate}

Greater $(P<0.05)$ arterial L-lactate concentrations (Table 1$)$ were measured when lucerne was meal-fed than when lucerne was timed-fed. Dietary regimen also affected VA for Llactate; VA were lower across PDV $(P<0.05)$ and stomach tissue $(P<0.1)$ when concentrate was given than when lucerne was given and were lower $(P<0.05)$ across MDV when lucerne was meal-fed than when lucerne was timed-fed. Net flux of L-lactate across PDV (Table 1) was lower $(P<0.05)$ when concentrate was given than when lucerne was given. This decrease in PDV L-lactate flux seems due to a decrease in appearance across stomach tissue, since MDV flux (Table 3$)$ was not affected by diet $(P>0.1)$ and stomach L-lactate VA were lower $(P<0.05)$ when concentrate was given. Stomach flux of L-lactate (Table 1) was not affected $(P>0 \cdot 1)$ by diet, although rates of absorption tended to be lower when concentrate was given and higher when lucerne was meal-fed. On average, MDV appearance of L-lactate accounted for $26 \%$ of PDV flux (Table 1).

Arterial L-lactate concentration increased 1.6-fold following meal-feeding of lucerne (linear $P<0.01$, quadratic $P<0.01$; Table 2). This substantial increase in arterial L-lactate concentration was associated with an increase in net PDV flux (quadratic $P<0.05$; Table 2), yet VA across stomach tissue decreased slightly (linear $P<0.01$ ) and increases in VA and net flux across MDV (Table 2$)$ were not significant $(P>0 \cdot 1)$. There were no significant effects of time $(P>0 \cdot 1)$ when concentrate was given (Table 3$)$.

\section{DISCUSSION \\ $V F A$}

Blood VFA concentrations in the present study were similar to those reported for dry Holstein cows determined using similar analytical techniques (Huntington \& Reynolds, 1983). Net rates of absorption of VFA across PDV in the present study were also comparable to those reported by Huntington \& Reynolds (1983) for dry Holstein cows consuming $5 \mathrm{~kg}$ dry matter/d and to rates reported for beef heifers on an identical concentrate diet at similar levels of intake (Huntington \& Prior, 1983). Ratios of the net absorption of acetate:propionate were also similar to previous measurements from cattle (Huntington \& Reynolds, 1983; Huntington \& Prior, 1983) and sheep (Bergman \& Wolff, 1971) fed on diets similar to those used in the present study.

On a net basis, stomach tissues accounted for $85-100 \%$ of net PDV absorption of VFA. Barcroft et al. (1944) measured significant VA for VFA in blood draining the rumen and reticulum. For blood draining the omasum and caecum, VA of VFA were half those measured for the reticulo-rumen. Our measurements of stomach net flux include the reticulo-rumen and omasum. The caecum is a component of the MDV; however, net absorption of VFA from this section of the gut must be relatively small compared with the absorption across stomach tissues, since it receives a much lower portion of PDV blood flow than stomach tissues (Schaefer \& Young, 1980).

There was net utilization of acetate across the MDV of steers given lucerne (Table 1), which agrees with the slightly negative VA for VFA across the small intestine of sheep reported by Barcroft et al. (1944). In sheep, about one-third of acetate in the arterial supply to the PDV is removed (Bergman \& Wolff, 1971). Pethick et al. (1981) found that acetate used by PDV of sheep was primarily oxidized and not incorporated into mesenteric fat. Both Bergman \& Wolff (1971) and Pethick et al. (1981) determined that PDV acetate use 
represented about $25 \%$ of whole-body acetate turnover in sheep fed on lucerne. The relative contribution of stomach and post-stomach tissues to total PDV arterial acetate utilization is not known. Calculations of Bergman \& Wolff (1971) and in vitro measurements of Stevens \& Stettler (1966) show that $30-45 \%$ of the acetate absorbed from the rumen may be metabolized by rumen tissues, but no measurements of arterial acetate utilization by stomach tissues have been reported. These studies also found that $50-65 \%$ of propionate and $85-90 \%$ of butyrate absorbed from the rumen are metabolized by rumen epithelium and never appear in the portal vein. Our measurements of net PDV absorption of VFA underestimate actual absorption of VFA from the gut lumen to the extent of their metabolism by epithelial tissues before entering venous blood. For acetate, net PDV flux measurements also underestimate actual absorption to the extent of arterial acetate utilization by PDV tissues. Bergman \& Wolff (1971) found net PDV flux measurements represented only $60-70 \%$ of actual acetate absorption.

Only arterial acetate concentrations fluctuated significantly during sampling. Masson \& Phillipson (1951) reported that in sheep, arterial acetate, but not propionate and butyrate concentrations, relate to absorption across PDV. This is due to differences in the capacity of hepatic tissues for metabolism of individual VFA. Bergman \& Wolff (1971) reported that $80-90 \%$ of the propionate and butyrate entering the liver were removed, while only $1-3 \%$ of acetate was removed from the hepatic circulation. The increase in arterial acetate concentration observed following meal-feeding of lucerne to steers in the present study was probably due to the concomitant postprandial increase in net PDV acetate absorption.

\section{$B O H B$}

In the fed ruminant, metabolism of absorbed butyrate to BOHB results in essentially all PDV ketone body production (Katz \& Bergman, 1969). Therefore, our measurements of PDV production of BOHB directly reflect amounts of absorbed butyrate from the gut lumen metabolized to BOHB. Assuming all BOHB was produced from the metabolism of absorbed butyrate and that the metabolism of butyrate to other compounds was negligible, net PDV absorption of $n$-butyrate represents $30 \%$ of actual absorption (BOHB net production $+n$-butyrate net absorption) when lucerne was given. This is somewhat higher than the $10-15 \%$ value given by Bergman \& Wolff (1971) for sheep fed on lucerne and may relate to conversion of $n$-butyrate to $\mathrm{CO}_{2}$ and other metabolites. Stevens \& Stettler (1966) reported that $75 \%$ of the butyrate metabolized by rumen epithelium in vitro was converted to BOHB.

In the present study essentially all net PDV production of BOHB was attributable to stomach tissues. In rats, Windmueller \& Spaeth (1978) found that arterial BOHB was a primary source of the carbon in $\mathrm{CO}_{2}$ produced across the jejunum. Our findings suggest that some utilization of arterial BOHB by PDV tissues also occurs in the bovine. Like acetate, BOHB removed across MDV tissues may be oxidized or incorporated into mesenteric fat. Katz \& Bergman (1969) reported net utilization of BOHB across the total PDV in fasted, twin-pregnant sheep. Net absorption of $n$-butyrate across MDV in our steers was relatively small, yet on average net $n$-butyrate absorption was greater than the net use of BOHB. In the caecum and large intestine of rabbits, $n$-butyrate is largely metabolized to $\mathrm{CO}_{2}$ rather than ketone bodies and represents an important energy substrate for hind-gut tissues (Marty \& Vernay, 1984). Pennington (1951) found that the conversion of $n$-butyrate to BOHB in vitro by caecal tissue from sheep was much lower than that for rumen tissue. One explanation for the absence of BOHB production across MDV in these steers is that $n$-butyrate absorbed from the hind-gut and metabolized is oxidized to $\mathrm{CO}_{2}$ rather than ketone bodies as in the rumen epithelium. Another explanation would be that BOHB production occurred across MDV, yet was slightly less than MDV 
use of arterial BOHB such that small negative net flux rates were measured. Regardless, on a net basis PDV production of BOHB appears to be attributable totally to the stomach tissues in beef steers.

Increases in arterial concentration of $\mathrm{BOHB}$ when concentrate $v$. lucerne was given (Table 1) were not associated with significant increases in PDV production (Table 3). While not measured in the present study, increased hepatic ketogenesis or decreased utilization or both, may have caused the increase in arterial concentration of BOHB with concentrate feeding. If butyrate uptake by hepatic tissues increased in response to increased PDV absorption of $n$-butyrate when concentrate was given (Table 1 ), then additional precursers for hepatic BOHB production would be available.

Increased absorption of $n$-butyrate with concentrate feeding could be due to changes in rumen VFA production (Thorlacius \& Lodge, 1973) or to increased $n$-butyrate absorption across the rumen epithelium due to lower rumen $\mathrm{pH}$. Decreased lumen $\mathrm{pH}$ increases $n$ butyrate absorption across the rumen epithelium in vitro, without increasing ketone body production (Stevens \& Stettler, 1966). In the present study, concentrate feeding increased net absorption of $n$-butyrate without affecting net BOHB production (Table 3 ). Increases in net absorption of $n$-butyrate were due to increases in VA across stomach tissues.

\section{L-lactate}

Arterial concentrations of L-lactate were similar to values measured in steers fed on identical diets by Huntington et al (1981), though net flux rates for PDV were somewhat higher in the present study. Our net L-lactate flux rates across PDV were lower than rates for heifers fed on the same concentrate diet at similar intakes (Huntington \& Prior, 1983). While Huntington et al. (1981) observed no change in PDV L-lactate appearance in steers switched from lucerne hay to concentrate, we observed a decrease in PDV appearance (Table 3). Janes et al. (1985) found net MDV L-lactate VA in sheep to be variable and often negative. In contrast, VA for L-lactate across MDV in the present study were positive for all but two of sixty observations and averaged $60.0 \%$ of VA for PDV. Portions of PDV Llactate production attributable to known sources (dietary lactate absorption and tissue metabolism of glucose and propionate) are not known. Studies by Weigand et al. (1972) and Weekes \& Webster (1975) suggest that the conversion of propionate to L-lactate in rumen epithelium is relatively small compared with the conversion of glucose to L-lactate. Findings of Janes et al. (1985) indicate that $42.5 \%$ of the L-lactate appearing across MDV of sheep results from glucose metabolism, which suggests that substantial glycolytic activity occurs in tissues of MDV. In vitro studies have shown that epithelial tissue from the entire length of the small intestine of sheep has a large capacity for the conversion of glucose to L-lactate (Wahle et al. 1972).

In conclusion, MDV tissues contribute relatively minor amounts to the net flux of VFA and BOHB across the total PDV of beef steers fed on lucerne or concentrate. Net utilization of acetate and BOHB was measured across MDV when lucerne was given to these steers. Feeding concentrate $v$. lucerne increased net PDV absorption of $n$-butyrate and $n$-valerate, decreased net PDV appearance of L-lactate and decreased net stomach absorption of 3methylbutyrate but had no effect on the relative contribution of MDV to total PDV flux.

The authors wish to express their appreciation to Dr P. J. Reynolds for conducting VFA analyses, J. Whitt, E. Zetina and B. Gadsen for their outstanding technical assistance, Dr B. Stroud and staff for surgical assistance and Animal Operations Personnel for assisting in animal care and feeding. 


\section{REFERENCES}

Barcroft, J., McAnally, R. A. \& Phillipson, A. T. (1944). Journal of Experimental Biology 20, 120-129.

Bergman, E. N. \& Wolff, J. E. (1971). American Journal of Physiology 221, 586-592.

Giesecke, D. \& Stangassinger, M. (1980), In Digestive Physiology and Metabolism in Ruminants, pp. 523-540. [Y. Ruckebush and P. Thivend, editors]. Lancaster: MTP Press.

Huntington, G. B. \& Prior, R. L. (1983). Journal of Nutrition 112, 2280-2288.

Huntington, G. B., Prior, R. L. \& Britton, R. A. (1981). Journal of Nutrition 111, 1164-1172.

Huntington, G. B. \& Reynolds, P. J. (1983). Journal of Dairy Science 66, 86-92.

Janes, A. N., Weekes, T. E. C. \& Armstrong, D. G. (1985). British Journal of Nutrition 54, 449-458.

Katz, M. L. \& Bergman, E. N. (1969). American Journal of Physiology 216, 953-960.

Marty, J. \& Vernay, M. (1984). British Journal of Nutrition 51, 265-277.

Masson, M. J. \& Phillipson, A. T. (1951). Journal of Physiology 113, 189-206.

Pennington, R. J. (1951). Biochemical Journal 51, 251-258.

Pethick, D. W., Lindsay, D. B., Barker, P. J. \& Northrup, A. J. (1981). British Journal of Nutrition 46, $97-110$.

Reynolds, C. K. \& Huntington, G. B. (1988). British Journal of Nutrition 60, 539-551.

Reynolds, P. J., Huntington, G. B. \& Reynolds, C. K. (1986). Journal of Animal Science 63, Abstr.

Schaefer, A. L. \& Young, B. A. (1980). Canadian Journal of Animal Science 60, 677-681.

Somogyi, M. (1945). Journal of Biological Chemistry 160, 69-73.

Stevens, C. E. \& Stettler, B. K. (1966). American Journal of Physiology 210, 365-372.

Thorlacius, S. O. \& Lodge, G. A. (1973). Canadian Journal of Animal Science 53, 279-288.

Wahle, K. W. J., Weekes, T. E. C. \& Sherratt, H. S. A. (1972). Comparative Biochemistry and Physiology 41B, $759-769$.

Weekes, T. E. C. \& Webster, A. J. F. (1975). British Journal of Nutrition 33, 345-438.

Weigand, E., Young, J. W. \& McGilliard, A. D. (1972). Biochemical Journal 126, 201-209.

Williamson, D. H. \& Mellanby, J. M. (1974). In Methods of Enzymatic Analysis, 2nd ed, pp. $1837-1839$ [E. N. Bergmeyer, editor]. New York: Academic Press.

Windmueller, H. G. \& Spaeth, A. E. (1978). Journal of Biological Chemistry 253, 69-76. 